FACTA UNIVERSITATIS

Series: Mechanical Engineering Vol. 15, No 1, 2017, pp. 73 - 83

DOI: 10.22190/FUME161013006M

Original scientific paper

\title{
A THEORETICAL-EXPERIMENTAL APPROACH FOR ELASTO-DAMPING PARAMETERS ESTIMATION OF CONE INERTIAL CRUSHER MOUNTING
}

\author{
UDC 622.7
}

\author{
Rosen Mitrev ${ }^{1}$, Simeon Savov ${ }^{2}$ \\ ${ }^{1}$ Technical University, Mechanical Engineering Faculty, Sofia, Bulgaria \\ ${ }^{2}$ Mining and Geology University, Sofia, Bulgaria
}

\begin{abstract}
The present paper deals with estimation of the elasto-damping parameters of a cone inertial crusher mounting. The numerical values of these parameters are crucial for accurate reproduction of the machine vibrational behavior and dynamical model adequacy. Due to the significant difficulties arising during the purely theoretical determination of the stiffness and damping parameters of the rubber vibroisolators it is well-suited to use a theoretical-experimental approach. The developed approach is based on the theoretical determination of the mounting stiffness parameters as a function of two experimentally measured natural frequencies of the mechanical system. The crusher is represented as a six degrees of freedom system with two planes of symmetry. By using the system characteristic polynomial, the theoretical derivation of mathematical relationships for the mechanical system natural frequencies as a function of stiffness, inertial and geometrical parameters is performed. A good agreement is shown when comparing the experimental and the theoretical results for the system kinematical characteristics.
\end{abstract}

Key Words: Cone Inertial Crusher, Modal Analysis, Parameters Estimation

\section{INTRODUCTION}

In recent years, in some cases of their application, the cone inertial crushers have been established as the only option for an effective and low energy milling of mineral raw materials [1]. The design feature that makes them particularly preferred is the absence of a rigid kinematical connection between the driving system and the internal crushing cone. That is why this type of crushers is protected against damage in the case of an unbreakable object's entering their crushing chamber.

Received October 13, 2016 / Accepted December 26, 2016

Corresponding author: Rosen Mitrev

Technical University, Mechanical Engineering Faculty, Kliment Ohridski 8 Blvd., Sofia, Bulgaria

E-mail: rosenm@tu-sofia.bg 
Fig. 1 shows the kinematical scheme of a cone inertial crusher. An electric motor (pos. 7), V-belt transmission (pos. 6), elastic rubber coupling (pos. 5), universal joint (pos. 4) and adjustable eccentric weight (pos. 3) realize the driving of the internal crushing cone (pos. 2). The outer crushing cone is rigidly mounted in the machine housing (pos. 1) placed on four rubber vibroisolators (pos. 8), rigidly connected to the ground. In the machine non-operating condition, the vertical axes of the outer and the internal cones and the eccentric weight axis of rotation are aligned, while in the operating mode this is not true due to the internal cone precession.

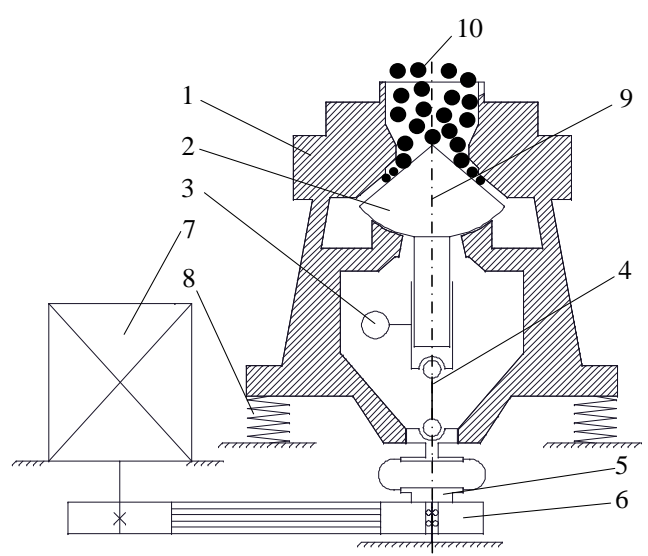

Fig.1 Kinematical scheme of the cone inertial crusher

Generated by the eccentric weight, the centrifugal force causes the internal cone to travel along the inner surface of the outer cone. The inertial force of the internal cone in addition to the inertial force of the eccentric weight generates a crushing force and as a result the two cones are pressed toward each other and the mineral material (pos. 10) is fragmented. The change of the rotational speed of the adjustable eccentric weight causes change of the crushing force and the crusher operational characteristics. When an unbreakable object enters the crushing chamber, the internal cone stops its movement, but the eccentric weight continues to rotate and thus the machine is protected against damage.

A general trend in the theoretical investigation of the cone inertial crushers is the study of their motions and kinematical characteristics through dynamical models that allow the mathematical description of the machine behavior in 3D space [2]. A crucial step during the dynamical models generation is the estimation of the numerical values of the elastodamping, geometrical, force and inertial parameters that provide the best match between the model and the real world machine behavior. Obviously, to achieve maximum accuracy, numerical values of the parameters should be set appropriately. There is a variety of methods for identification of the mechanical dynamical systems and estimation of their parameters values $[3,4,5]$, a significant part of which are based on the study of the system properties in the time or frequency domain.

While the geometrical and inertial parameters of the machine are determined relatively easily by use of CAD models, direct measurements or calculations, the estimation of the mounting elasto-damping parameters is accompanied by significant difficulties. The results, received by use of theoretical relationships or computer simulation are unreliable, 
especially when the machine mounting is realized by rubber vibroisolators. Typically, the characteristics of the rubber vibroisolators are in general: nonlinear [6], changing over time due to ageing [7], dependant on the temperature [8] and vibration frequency [9] or are described by complex models depending on a set of experimentally determined constants [10]. In the engineering practice, the problem is overcome by the use of appropriately organized experimental research for elasto-damping parameters estimation. One commonly used method [11] is the experimental measurement of the most important characteristics of any mechanical oscillating system - the values of the natural frequencies and the subsequent estimation of the rigid body properties and stiffness parameters by the use of theoretical relationships derived from the conventional modal analysis of the system.

The main goal of the present paper is to develop and validate a theoretical-experimental approach for estimation of elasto-damping parameters of the cone inertial crusher mounting. For this purpose, we should obtain theoretical relationships that relate stiffness, geometrical and inertial parameters of the system with natural frequencies that can be easily determined experimentally.

The present paper is structured as follows: Section 1 comprises analyzed papers concerning some problems of estimation of the mounting system parameters and the goal of the paper is defined; Section 2 presents a developed dynamical model of the crusher which takes into account the geometrical and inertial symmetry of the system; Section 3 is devoted to the theoretical investigation of the oscillating system and development of mathematical relationships for natural frequencies of the system. Experimental setup, based on industrial crusher of type KID-300 and experimental measurements are presented in Section 4. Section 5 presents the results of the developed approach together with validation. Section 6 comprises the conclusion.

\section{DYNAMICAL MODELING}

For the purposes of the present study, the cone inertial crusher is represented in 3D space as a rigid body with $6 \mathrm{DOF}$ (pos. 1), mounted on four fixed to the ground similar rubber vibroisolators (pos. 2), see Fig. 2 a) and b)). The crusher has two vertical planes of geometrical and inertial symmetry and the vibroisolators are mounted symmetrically according to these planes - Fig. 2 b). Every vibroisolator is represented by a set of three linear elasto-damping elements whose directions are aligned with the global coordinate system axes. To the ground is connected a global immovable Cartesian coordinate system $O x y z$ and to the rigid body gravity center is fixed a Cartesian coordinate system Cxyz whose axes are principal axes of inertia of the body. In the static equilibrium, position origins $O$ and $C$ of the coordinate systems are coincident and the vector of generalized coordinates measured according to the equilibrium position is:

$$
\mathbf{q}=\left[\begin{array}{llllll}
x & y & z & \psi & \theta & \varphi
\end{array}\right]^{T}
$$

where by $x, y$ and $z$ are denoted the linear translations of body mass center $\mathrm{C}$, and by $\psi, \theta$ and $\varphi$ are denoted body rotations according to the corresponding axes, see Fig. 2. 


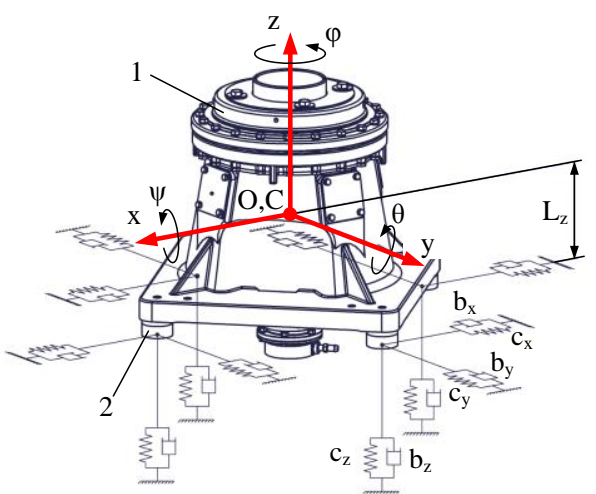

a)

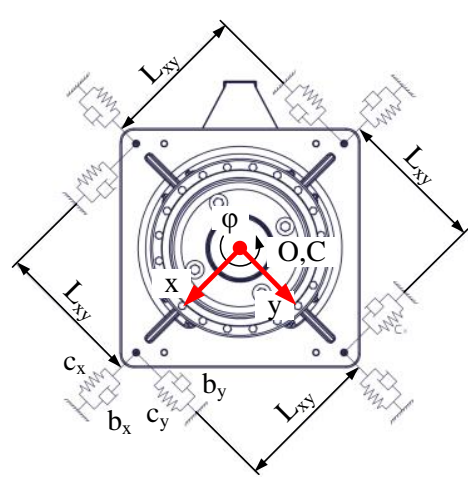

b)

Fig. 2 Dynamical model of the crusher: a) 3D view, b) top view

Undamped free vibrations of the system are represented by the following linear system of homogeneous ordinary differential equations of second order:

$$
\mathbf{M} \ddot{\mathbf{q}}+\mathbf{C q}=0
$$

where $\mathbf{M}$ is the mass matrix of the system, $\mathbf{C}$ is the stiffness matrix, and by $\ddot{\mathbf{q}}=\left[\begin{array}{llllll}\ddot{x} & \ddot{y} & \ddot{z} & \ddot{\psi} & \ddot{\theta} & \ddot{\varphi}\end{array}\right]^{T}$ is denoted the vector of generalized accelerations.

Kinetic $T$ and potential $P$ energies of the system are positive definite quadratic forms of the correspondingly generalized velocities $\dot{\mathbf{q}}=\left[\begin{array}{llllll}\dot{x} & \dot{y} & \dot{z} & \dot{\psi} & \dot{\theta} & \dot{\varphi}\end{array}\right]^{T}$ and generalized coordinates Eq. (1):

$$
\begin{gathered}
T=\frac{1}{2} \dot{\mathbf{q}}^{\mathbf{T}} \mathbf{M} \dot{\mathbf{q}} \\
P=\frac{1}{2} \mathbf{q}^{\mathbf{T}} \mathbf{C q}
\end{gathered}
$$

Under assumption of small motions, the elements of mass matrix $\mathbf{M}$ and stiffness matrix $\mathbf{C}$ are computed as:

$$
\begin{gathered}
m_{i, j}=\frac{\partial^{2} T}{\partial \dot{q}_{i} \partial \dot{q}_{j}} \\
c_{i, j}=\frac{\partial^{2} P}{\partial q_{i} \partial q_{j}}
\end{gathered}
$$

Performing the differentiations in Eqs. (5) and (6) and taking into account the geometrical and mass symmetry of the system, for the mass and stiffness matrix we obtain [2]:

$$
\mathbf{M}=\operatorname{diag}\left(m, m, m, J_{x y}, J_{x y}, J_{z}\right)
$$




$$
\mathbf{C}=\left[\begin{array}{cccccc}
4 c_{x y} & 0 & 0 & 0 & -4 c_{x y} L_{z} & 0 \\
0 & 4 c_{x y} & 0 & 4 c_{x y} L_{z} & 0 & 0 \\
0 & 0 & 4 c_{z} & 0 & 0 & 0 \\
0 & 4 c_{x y} L_{z} & 0 & 2 L_{x y}^{2} c_{z}+4 L_{z}^{2} c_{x y} & 0 & 0 \\
-4 c_{x y} L_{z} & 0 & 0 & 0 & 2 L_{x y}^{2} c_{z}+4 L_{z}^{2} c_{x y} & 0 \\
0 & 0 & 0 & 0 & 0 & 4 L_{x y}^{2} c_{x y}
\end{array}\right]
$$

In Eqs. (7) and (8) are used the following notations: $m$ - mass of the crusher housing (pos.1, Fig.1) together with the contained movable elements (pos. 2, 3, 4 in Fig. 1); $J_{x}=J_{y}=J_{x y}$ and $J_{z^{-}}$the mass moments of inertia of the crusher according to coordinate system $C x y z$ axes; $c_{x}=c_{y}=c_{x y}$ and $c_{z}$ - stiffness coefficients of vibroisolators in $x, y$ and $z$ directions; $L_{z}$ - distance in $z$ direction between the gravity center and the point of connection of the vibroisolator to the ground; $L_{x y}$ - distance in $x$ and $y$ directions between the gravity center and the point of connection of the vibroisolator to the ground.

\section{Determination of the System Natural FreQuencies AND Mode ShapeS}

Matrices $\mathbf{M}$ and $\mathbf{C}$ can be exploited to determine dynamical matrix $\mathbf{H}$ of the system and corresponding characteristic polynomial $f(\lambda)[12]$ :

$$
\begin{gathered}
\mathbf{H}=\mathbf{M}^{-1} \mathbf{C} \\
f(\lambda)=\operatorname{det}(\mathbf{H}-\lambda \mathbf{I})
\end{gathered}
$$

where $\mathbf{I}$ is the identity matrix.

After performing mathematical operations in Eqs. (9) and (10) for the characteristic polynomial we obtain:

$$
f(\lambda)=\left(\lambda m-4 c_{z}\right)\left(\lambda J_{z}-4 L_{x y}^{2} c_{x y}\right)\left(\begin{array}{l}
\lambda m\left(\lambda J_{x y}-2 L_{x y}^{2} c_{z}\right)-\ldots \\
\ldots-4 c_{x y}\left(\lambda\left(J_{x y}+m L_{z}^{2}\right)-2 L_{x y}^{2} c_{z}\right)
\end{array}\right)^{2}
$$

Roots $\lambda_{i}$ of characteristic polynomial $f(\lambda)$ are the eigenvalues of matrix $\mathbf{H}$ and are equal to squared natural frequencies $\omega_{i}$ of the mechanical system under consideration:

$$
\begin{gathered}
\lambda_{1}=\lambda_{2}=\left(\omega_{1}^{x}\right)^{2}=\left(\omega_{2}^{y}\right)^{2}=\frac{k_{1}-k_{2}}{m J_{x y} J_{z}} \\
\lambda_{3}=\left(\omega_{3}^{z}\right)^{2}=\frac{4 c_{z}}{m} \\
\lambda_{4}=\lambda_{5}=\left(\omega_{4}^{\psi}\right)^{2}=\left(\omega_{5}^{\theta}\right)^{2}=\frac{k_{1}+k_{2}}{m J_{x y} J_{z}} \\
\lambda_{6}=\left(\omega_{6}^{\varphi}\right)^{2}=\frac{4 L_{x y}^{2} c_{x y}}{J_{z}}
\end{gathered}
$$


where: $k_{1}=J_{z}\left(2 c_{x y}\left(J_{x y}+m L_{z}^{2}\right)+m L_{x y}^{2} c_{z}\right) \quad, k_{2}=\sqrt{J_{z}^{2}\left(\begin{array}{l}\left.\left(2 c_{x y}\left(J_{x y}+m L_{z}^{2}\right)+m L_{x y}^{2} c_{z}\right)^{2}-\ldots\right) \\ \ldots-8 m L_{x y}^{2} c_{x y} c_{z} J_{x y}\end{array}\right)}$, and $\omega_{1}^{x}, \omega_{2}^{y}, \omega_{3}^{z}, \omega_{4}^{\psi}, \omega_{5}^{\theta}, \omega_{6}^{\varphi}$ are the natural frequencies associated to the corresponding coordinates. As can be seen from Eqs. (12) and (14), due to the symmetry of the system there are two repeated roots and that is why the system has only four unique natural frequencies.

Eigenvectors $\mathbf{u}_{\mathbf{i}}$ corresponding to natural frequencies $\omega_{i}$ are determined from the following equation:

$$
\left(\mathbf{H}-\lambda_{i} \mathbf{I}\right) \mathbf{u}_{i}=0
$$

Modal matrix $\boldsymbol{\Phi}$ of the system is composed of the eigenvectors

$$
\boldsymbol{\Phi}=\left[\begin{array}{llllll}
\mathbf{u}_{1} & \mathbf{u}_{2} & \mathbf{u}_{3} & \mathbf{u}_{4} & \mathbf{u}_{5} & \mathbf{u}_{6}
\end{array}\right]
$$

and for the real world machine parameters take the form:

$$
\boldsymbol{\Phi}=\left(\begin{array}{cccccc}
-0.93 & 0 & 0 & 0 & 0.03 & 0 \\
0 & -0.93 & 0 & -0.03 & 0 & 0 \\
0 & 0 & 1 & 0 & 0 & 0 \\
0 & 0.38 & 0 & -1 & 0 & 0 \\
-0.38 & 0 & 0 & 0 & -1 & 0 \\
0 & 0 & 0 & 0 & 0 & 1
\end{array}\right)
$$

The review of the modal matrix shows that the translation along $z$ axis and rotation around $z$ axis are uncoupled while the translations along $x$ and $y$ coordinates and rotations around $y$ and $x$ axes are coupled.

Providing experimentally measured values for two unique natural frequencies and knowing the inertial and geometrical parameters of system, Eqs. (12-15) can be used for determination of unknown stiffness parameters $c_{x y}$ and $c_{z}$. A total of six combinations in pairs are available for four unique natural frequencies, namely combinations $\left(\omega_{1}^{x}, \omega_{3}^{z}\right)_{1}$, $\left(\omega_{1}^{x}, \omega_{4}^{\psi}\right)_{2},\left(\omega_{1}^{x}, \omega_{6}^{\varphi}\right)_{3},\left(\omega_{3}^{z}, \omega_{4}^{\psi}\right)_{4},\left(\omega_{3}^{z}, \omega_{6}^{\varphi}\right)_{5},\left(\omega_{4}^{\psi}, \omega_{6}^{\varphi}\right)_{6}$. After the determination of the values of natural frequencies of a particular combination, using Eqs. (12-15) the corresponding system of equations can be solved analytically or numerically for unknown stiffness parameters. Least computational complexity has combination $\left(\omega_{3}^{z}, \omega_{6}^{\varphi}\right)_{5}$ for which from Eqs. (13) and (15) we obtain:

$$
\begin{gathered}
c_{z}=\frac{m\left(\omega_{3}^{z}\right)^{2}}{4} \\
c_{x y}=\frac{J_{z}\left(\omega_{6}^{\theta_{z}}\right)^{2}}{4 L_{x y}^{2}}
\end{gathered}
$$

Due to the large weight of the machine and its design features, a particular problem in using this combination are the difficulties during excitation of oscillations along $z$-axis and around $z$-axis. The easiest way to excite oscillations is along axis $x$ (or $y$ ) and the 
coupled oscillations around $y$ (or $x$ ) axis and thus values of the natural frequencies for second combination $\left(\omega_{1}^{x}, \omega_{4}^{\psi /}\right)_{2}$ can be determined. These values along with the Eqs. (12) and (14) can be used for calculation of $c_{x y}$ and $c_{z}$. The system has two solutions and due to the vibroisolator design, one must choose the solution for which is fulfilled $c_{z}>c_{x y}$ :

$$
\begin{gathered}
c_{z}=\frac{n_{1}+n_{2}}{4 L^{2} m} \\
c_{x y}=\frac{n_{1}-n_{2}}{4 L^{2} m}
\end{gathered}
$$

where $n_{1}=m\left(\omega_{4}^{\psi}+\omega_{1}^{x}\right)^{2} J_{x y}, n_{2}=\sqrt{m^{2} J_{x y}\left(J_{x y}\left(\left(\omega_{4}^{\psi \prime}\right)^{2}-\left(\omega_{1}^{x}\right)^{2}\right)^{2}\right)-4 m\left(\omega_{4}^{\psi}\right)^{2}\left(\omega_{1}^{x}\right)^{2} L_{z}^{2}}$.

\section{EXPERIMENTAL SETUP AND MEASUREMENTS}

For determination of natural frequencies $\omega_{1}^{x}$ and $\omega_{4}^{\psi}$ an experimental study is conducted. The used experimental setup (Fig. 3) is based on the industrially used cone inertial crusher of type KID-300. The numerical values of the experimental setup parameters are shown in Table 1.

Table 1 Numerical values of the experimental setup parameters

\begin{tabular}{ll}
\hline Parameter & Value and dimension \\
\hline Mass of the crusher vibrating parts & $m=838.6 \mathrm{~kg}$ \\
Inertial moments & $J_{x y}=61.44 \mathrm{~kg} . \mathrm{m}^{2}, J_{z}=51.52 \mathrm{~kg} . \mathrm{m}$ \\
Distances & $L_{x y}=0.486 \mathrm{~m}, L_{z}=0.3 \mathrm{~m}, \mathrm{~L}=0.212 \mathrm{~m}$ \\
Mass of the eccentric weight & $m_{v}=43.1 \mathrm{~kg}$ \\
Radius of rotation of the eccentric weight & $e=0.03 \mathrm{~m}$ \\
Angular velocity of the eccentric weight & $\omega=113 \mathrm{rad} / \mathrm{s}, 157 \mathrm{rad} / \mathrm{s}$ \\
\hline
\end{tabular}

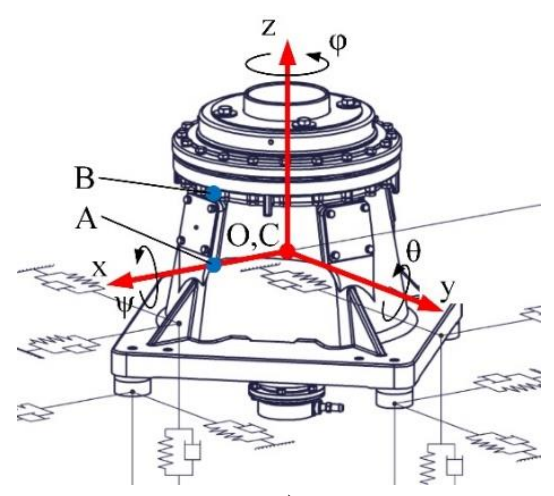

a)

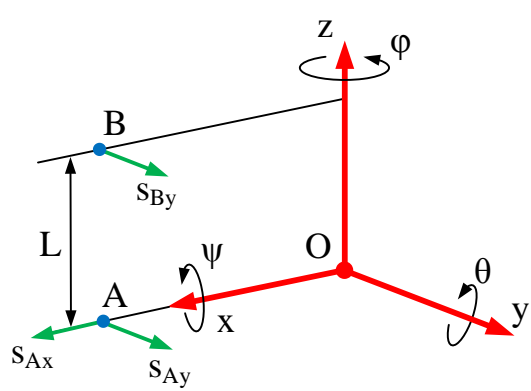

b)

Fig. 3 Scheme of the experimental setup and accelerometers layout 
In Fig. 3 a) and b) the mounting positions of the used piezoelectric accelerometers are shown. At point A, situated on the housing and on local $x$ axis, is mounted one two-axial accelerometer whose axes are directed along $x$ and $y$ axes of the local coordinate system Fig. $3 \mathrm{~b}$ ). The second accelerometer is uniaxial and is mounted also on the housing in point B situated above point A. Its axis is directed along local $y$ axis. Accelerometermeasured linear accelerations $s_{A x}, s_{A y}$ and $s_{B y}$ are used for calculation of the generalized accelerations along coordinates $x$ and $\psi$ under the small motion assumption:

$$
\begin{gathered}
\ddot{x}=s_{A x} \\
\ddot{\psi}=\frac{s_{B y}-s_{A y}}{L}
\end{gathered}
$$

The vibration signal captured for 4 seconds which occurred as a result of an artificial impulse excitation along $x$ axis is shown in Fig. 4 a). In the same Fig. the signal after the high frequency filtering is shown. The value of natural frequency $\omega_{1}^{x}$ is determined as shown in Fig. 4 b) FFT spectral analysis of the vibration signal. Similarly, two other signals are captured and according to Eq. (24) the angular acceleration is computed and natural frequency $\omega_{4}^{\psi}$ is determined. The received values for the two frequencies are $\omega_{1}^{x}=17.71 \mathrm{rad} / \mathrm{s}$ and $\omega_{4}^{\psi}=64.10 \mathrm{rad} / \mathrm{s}$.

Additionally, the filtered acceleration signal, shown in Fig. 4 a) is used to determine vibroisolator damping parameter $b_{x}$. Exponentially decaying consecutive amplitudes of the acceleration suggest predominantly viscous damping in the system and asymptotically stable motion [13]. In Fig. 5 the filtered acceleration signal and decaying exponential curve, which is a regression line for amplitudes peak points, are shown. The exponential curve is described by Eq. (25), where the constants determined by the least-squares method are $A_{0}=1.209 \mathrm{~m} / \mathrm{s}^{2}$ and $n=-0.804$.

$$
\ddot{x}_{e}=A_{0} e^{-n t}
$$

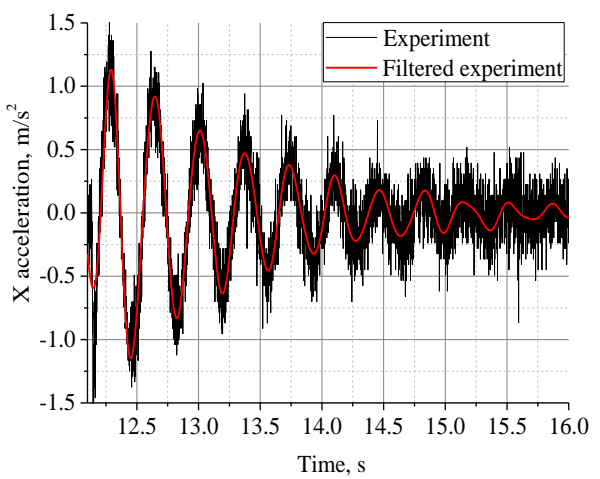

a)

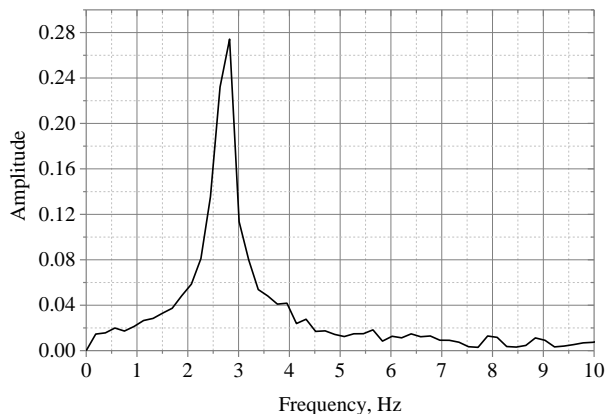

b)

Fig. 4 Measured vibration signal a) and spectral analysis b) 


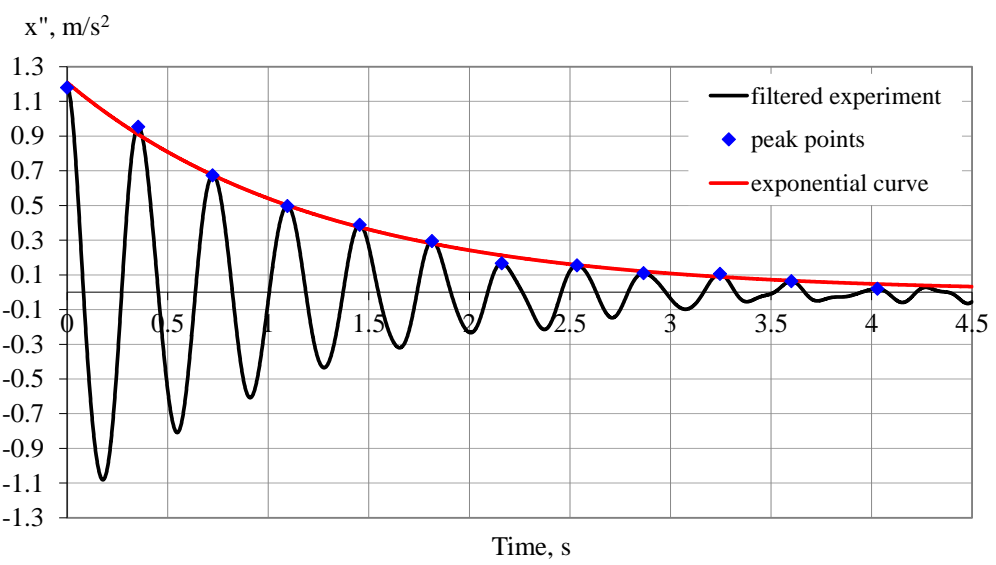

Fig. 5 Filtered acceleration signal and exponential curve

If along $x$ axis the system is considered as that of a single degree of freedom, then the total for all four vibroisolators damping coefficient is determined as:

$$
b_{x y}^{\Sigma}=2 m n
$$

and its value is $b_{x y}^{2}=1348.8 \mathrm{Ns} / \mathrm{m}$ and correspondingly, the damping coefficient for one vibroisolator is $b_{x y}=337.2 \mathrm{Ns} / \mathrm{m}$. Similarly, the measured value for damping coefficient in $z$ direction is $b_{z}=1039 \mathrm{Ns} / \mathrm{m}$.

\section{NUMERICAL EXAMPLE AND DISCUSSION}

To illustrate and validate the developed approach, the stiffness parameters of the experimental setup mounting are determined. If the already experimentally determined values of natural frequencies $\omega_{1}^{x}=17.71 \mathrm{rad} / \mathrm{s}$ and $\omega_{4}^{\psi}=64.10 \mathrm{rad} / \mathrm{s}$ are substituted in Eqs. (21) and (22) then the values of the stiffness coefficients are computed as $c_{z}=472.3 \mathrm{kN} / \mathrm{m}$ and $c_{x y}=74.4 \mathrm{kN} / \mathrm{m}$ for a single vibroisolator. According to Eqs. (13) and (15) the stiffness values are used for computation of the two other unique natural frequencies. The computed values are $\omega_{3}^{z}=47.46 \mathrm{rad} / \mathrm{s}$ and $\omega_{6}^{\varphi}=36.93 \mathrm{rad} / \mathrm{s}$ which are very close to the experimentally measured values $46.60 \mathrm{rad} / \mathrm{s}$ and $36.91 \mathrm{rad} / \mathrm{s}$ correspondingly.

To validate the dynamical model and check the reliability of the estimated values of the stiffness and damping parameters additional experimental measurements are carried out. The system excitation is performed by the generated due rotation of the eccentric weight force - pos. 3, Fig. 1. In this case, the eccentric weight rotation causes a harmonic excitation and the resultant motion of the system is described by the following system of equations:

$$
\mathbf{M} \ddot{\mathbf{q}}+\mathbf{B} \dot{\mathbf{q}}+\mathbf{C q}=\mathbf{F}
$$

where $\mathbf{B}$ is the damping matrix, received in a formal way by replacing symbol " $c$ " in $\mathbf{C}$ by symbol " $b$ ", and $\mathbf{F}$ is a vector of generalized forces: 


$$
\mathbf{F}=\left[\begin{array}{llllll}
F_{x} & F_{y} & 0 & 0 & 0 & 0
\end{array}\right]^{T}
$$

In Eq. (28) $F_{x}$ and $F_{y}$ denote the harmonic excitation forces generated by the eccentric weight rotation:

$$
\begin{aligned}
& F_{x}=m_{v} \omega^{2} e \sin (\omega t) \\
& F_{y}=m_{v} \omega^{2} e \cos (\omega t)
\end{aligned}
$$

where $m_{v}$ is the eccentric weight mass, $e$ is eccentricity of the weight, $\omega$ is the eccentric weight angular velocity.

In Fig. 5 the captured experimental vibration signal is shown as well as the numerical solution for acceleration of system of Eqs. (27) in which the determined values of the stiffness and damping coefficients are used.

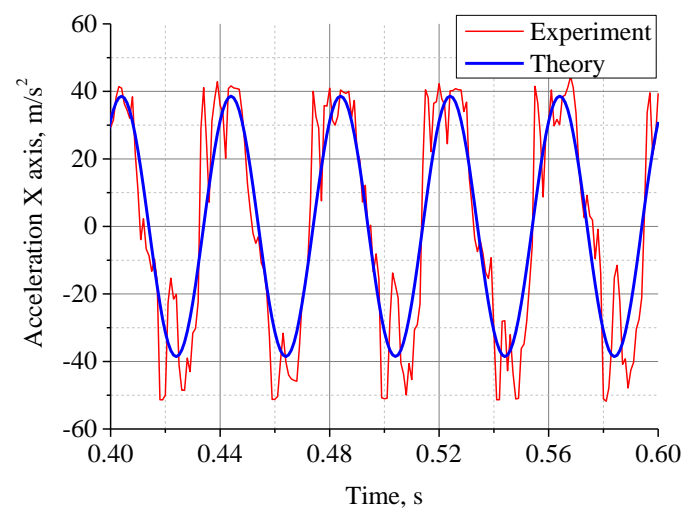

a)

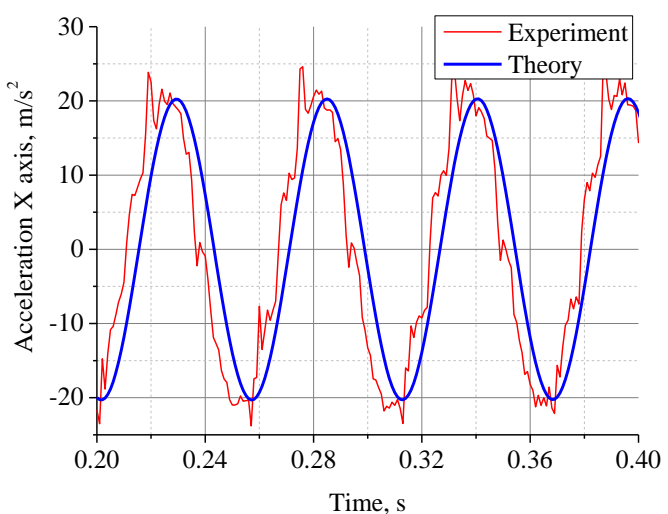

b)

Fig. 6 Graphs of numerical and experimental vibration signal:

a) $\omega=157 \mathrm{rad} / \mathrm{s}$, b) $\omega=113 \mathrm{rad} / \mathrm{s}$ 
The experiments are performed for two typical values of the angular velocities in the crusher operating range $-\omega=157 \mathrm{rad} / \mathrm{s}$ and $\omega=113 \mathrm{rad} / \mathrm{s}$. As can be seen, the experimental and numerical results show good agreement both in amplitude and in frequency and this is confirmation of the determined values correctness and adequacy of the dynamical model.

\section{CONCLUSION}

The significant difficulties arising during the purely theoretical estimation of the elasto-damping parameters of the cone inertial crusher mounting can be successfully overcome by using the developed theoretical-experimental approach. The single performed experiment allows - by capturing vibration signals of the suitable mounted one two-axial and one uniaxial accelerometers - to determine two unique natural frequencies of the crusher. By using Eqs. (12-15), Eq. (21) and Eq. (22) along with measured natural frequencies, the stiffness parameters and two other unique frequencies can be calculated. Because of good agreement has been shown when comparing the experimental and theoretical results it can be concluded that the developed approach is suitable for estimation of the elasto-damping parameters of a cone inertial crusher mounting. The approach could be easily applied to other mechanical structures with two planes of symmetry according to the vertical planes.

\section{REFERENCES}

1. Blazy P., Zarogatsky L., Jdid E., 1994, Hamdadou M., Vibroinertial comminution — principles and performance, International Journal of Mineral Processing, 41(1-2), pp.33-51.

2. Savov S., 2014, Research of mechanical and technological parameters of cone inertial crushers type KID, Ph.D thesis, Mining and Geology University "St. Ivan Rilski”, Sofia, 221 p.

3. Savov S., 2016, Experimental determination of natural frequencies of cone inertial crusher (KID-300), Bulgarian journal for engineering design, issue 30, october 2016, pp.5-10.

4. Ljung L., 1999, System Identification: Theory for the User, Englewood Cliffs, NJ: Prentice-Hall, 672 p.

5. Grigorov B., Mitrev R., 2016, Dynamic behavior of a hydraulic crane operating a freely suspended payload, J Zhejiang Univ-Sci A, in press, doi: 10.1631/jzus.A1600292

6. Bruns J.-U., Lindner M., Popp, K., 2003, Identification of the Nonlinear Restoring Force Characteristic of a Rubber Mounting, Proc. Appl. Math. Mech., 2, pp. 270-271. doi:10.1002/pamm.200310120

7. Dashevskij M., Motorin V., Mironov E., Samoilenko T., 2003, Engineering design of rubber pads ageing properties: theory and experiment, Constitutive models for rubber III, Proceedings of the third European conference on constitutive models for rubber, 15-17 September 2003, London, UK.

8. Tachibana, E., Li, K., 1996, Temperature dependence of high damping rubber in base-isolated structures, Proceedings of the $11^{\text {th }}$ World Conference on Earthquake Engineering, Acapulco, Mexico. Paper no.492. Oxford: Pergamon.

9. Koblar, D., Boltezar, M., 2013, Evaluation of the frequency-dependent Young's modulus and damping factor of rubber from experiment and their implementation in a finite-element analysis, Experimental Techniques, published online 11 November 2013, doi: 10.1111/ext.12066

10. Feng, W., Hallquist, J., 2012, On Mooney-Rivlin Constants for Elastomers, $12^{\text {th }}$ International LS-DYNA Users Conference 2012, pp.1-10.

11. Malekjafarian A., Ashory R.M., Khatibi N.M., Latibari M.S., 2011, A new method for estimation of rigid body properties from output-only modal data, International Operational Modal Analysis Conference, IOMAC'11.

12. Strommen E., 2014, Structural Dynamics, Springer Series in Solid and Structural Mechanics, vol. 2, 510 p., doi: 10.1007/978-3-319-01802-7

13. Meirovitch L., 2001, Fundamentals of Vibrations, McGraw-Hill Higher Education, New York, 806 p. 